\title{
The effect of polar projection on the perception of euclidean structure from motion
}

\author{
ERIK BÖRJESSON and MATS LIND \\ Uppsala University, Uppsala, Sweden
}

\begin{abstract}
The aim of the present study was to examine whether euclidean structure could be recovered from apparent motion sequences under polar projection. In Experiment 1, length judgments of two sides of a simulated triangle rotating in depth did not reveal effects of type of projection, polar or parallel, on the perception of euclidean structure. However, there was a significant correlation between simulated and produced slants. The results also indicated that absolute depth judgments could not be accounted for by a random mechanism suggested by Todd and Bressan (1990). Experiments 2 and 3, in which a continuous dot surface was substituted for the triangle, showed that polar projection information from a relatively large visual angle, $17.40^{\circ}$, as compared with a small visual angle, $4.35^{\circ}$, facilitated discrimination of depth. Produced height:width ratios were consistently related to simulated shape, although the depth dimension was underestimated. Finally, Experiment 4 showed significant correlations between simulated and produced height:width ratios that could be accounted for only by an analysis in which $X$ and $Y$ velocities were treated independently. As in previous experiments, the variation in the depth dimension was underestimated. It was concluded that the visual system utilizes the additional information that is available in polar projection when recovering structure from motion, but that for different reasons the perceived structure does not become euclidean. These reasons are discussed briefly.
\end{abstract}

Ever since Wallach and O'Connell (1953) presented their results on the kinetic depth effect, a great deal of interest among psychologists has been directed to twodimensional (2-D) motion as providing information about 3-D space. This issue, currently often referred to as structure from motion, has been studied from different theoretical positions and with different methods. In particular, the studies have centered on the minimum conditions in the 2-D motion pattern needed for the reconstruction of 3-D space (e.g., see Braunstein, Hoffman, \& Pollick, 1990). Generally, the number of moving points and the number of views have been brought to a minimum. For instance, assuming a rigid 3-D object, it has been shown that three views and four noncoplanar points are sufficient mathematically for the reconstruction of the 3-D object (Ullman, 1979). Several analyses have been made with additional assumptions, for instance rotation with constant speed and fixed axis (see, e.g., Braunstein, Hoffman, Shapiro, Andersen, \& Bennett, 1987, for a review). In general, the analyses of minimum conditions have been made with parallel projection rather than with polar projection, because at small visual angles the 2-D differences between the two types of projections are very small. However,

This research was supported by grants from the Swedish Council for Research in the Humanities and Social Sciences. The authors are indebted to Eva Olsson, Ulf A.hlström, and Leif Eriksson for valuable assistance. We also want to thank James Todd and an anonymous reviewer for valuable comments on an earlier draft of the manuscript. Correspondence should be directed to E. Börjesson, Dept. of Psychology, Uppsala University, Box 1854, S-751 48 Uppsala, Sweden (e-mail: erik.borjesson@,psyk.uu.se). some objects in the real world are perceived under large visual angles. Likewise, the situation of a moving visual system creates an optic flow over the whole visual field. In both cases, an analysis of the 2-D-3-D correspondence in terms of parallel projection would be less appropriate. It should also be noted that minimum conditions for the reconstruction of 3-D differ between the two types of projections; two views under parallel projection will never be sufficient for a unique interpretation of a 3-D object (Bennett, Hoffman, Nicola, \& Prakash, 1989), whereas a unique interpretation of a rigid 3-D object can be obtained from two views under polar projection (e.g., LonguetHiggins, 1981).

Needless to say, human observers do not generally behave as "ideal" observers would. Several authors have therefore questioned the prevalent assumption of 3-D rigidity (e.g., Braunstein \& Andersen, 1986; Todd, 1985). Todd and his co-workers (Todd \& Bressan, 1990; Todd \& Norman, 1991) have recently argued that human observers are unable to reconstruct the euclidean properties of 3-D objects from apparent motion. Using parallel projections, they have shown that observers utilize, only to a very limited extent, more information than is present in two views. As a consequence, observers cannot completely reconstruct objects in 3-D space, because not all parameters can be estimated from only two views under parallel projection. The value of one parameter will remain unknown. This unknown value can be interpreted as a stretching factor along the line of sight and, because it is unknown, the exact (euclidean) 3-D shape of objects cannot be perceived. In light of this, Todd and his co-workers (Todd \& Bressen, 1990; Todd \& Norman, 1991) and 
Koenderink and van Doorn (1991) have proposed that, from motion, the visual system only records properties of 3-D objects that are invariant under affine transformations, disregarding properties that are invariant under only Euclidean transformations. The former properties are often referred to as "affine structure," the latter, as "euclidean structure."

If only two views at a time are used by the visual system, there is only one way to obtain information about the euclidean structure of a viewed object, and that is to use the unique information available in polar projections. The recovery of structure from two views under polar projections has been studied by several authors (e.g., Doner, Lappin, \& Perfetto, 1984; Lappin, Doner, \& Kottas, 1980; Lappin \& Fuqua, 1983). However, as pointed out by Todd and Bressan (1990), the judgments asked for in these studies, judgments including discrimination of rigid and nonrigid 3-D objects, do not necessarily require a correct interpretation of euclidean space. Therefore, it is not clear whether the observers exploited the information about euclidean structure provided by polar projection.

Our purpose in the present study was to explore this issue. In Experiment 1, the possibility of the perception of euclidean structure was studied by using a simulated triangle oscillating in depth and projected under polar or parallel projection. Because no euclidean percepts were recorded in Experiment 1, the same problem was readdressed in Experiment 2 with stimuli that were thought to be more compelling--smooth surfaces and displayed under larger visual angles. Reliable effects of visual angle were obtained, although the percepts were not strictly euclidean. In Experiment 3, dot density that covaried with visual angle was checked. Finally, in Experiment 4, a new test of the effect of polar information was performed by independently varying dot velocities parallel and at right angles with the simulated axis of rotation. Again, dot velocities parallel with the axis of rotation, which is information exclusive to polar projection, systematically affected perceived structure, although euclidean percepts were not obtained. Summarizing the results, it was concluded that polar projection did not yield perceived euclidean structure from motion. However, information in polar projection facilitated consistent relations between simulated and perceived depth.

\section{EXPERIMENT 1}

The main aim of Experiment 1 was to explore the possibility of euclidean space perception from motion by comparing the perception of distances and slants in depth when either polar or parallel projection was used. A second aim was to study the possibility of a random mechanism that could account for judgments of distances from motion, as suggested by Todd and Bressan (1990).

Todd and Bressan (1990) and Todd and Norman (1991) used only parallel projections. Although they rejected the notion that euclidean space was recovered, they never- theless found that the observers were able to make discriminations that could not fully be explained by an affine analysis. Even in cases with only two views, there were reliable effects of distances and angle of rotation of the 3-D object, tw o characteristics that cannot be differentiated from only two views under parallel projection. Todd and Bressan (1990) discussed this problem and proposed an ad hoc explanation according to which the judgments of length could be mediated by a specific value (c) denoting the rotary displacement in depth. From their data Todd and Bressan (1990) found that a random rectangular distribution of $1 / \varepsilon$, ranging from just above zero to 80 radians $^{-1}$, made the best fit to their obtained length judgments.

Experiment 1 showed simulated outline triangles rotating in depth according to either parallel or polar projection. To ascertain possible euclidean percepts, the subjects had to judge the relative lengths of two of the triangle's sides and its slant in depth. The latter measure also permitted an indirect way to evaluate the plausibility of Todd and Bressan's (1990) suggestion of the rectangular distribution of $1 / \varepsilon$ values.

\section{Method}

Apparatus and viewing conditions. The stimulus patterns were displayed by a monochrome Apple monitor, Type MO 400, with a display area of $21.3 \times 15.8 \mathrm{~cm}$ and a resolution of $77 \mathrm{dpi}$. Each pixel at the center of the screen covered a visual angle of $2^{\prime}$. The patterns were presented through a black tunnel attached to the computer screen. The length of the tunnel was $57.8 \mathrm{~cm}$. At the subject's end, there was a small aperture (with a radius of $1 \mathrm{~cm}$ ) in the tunnel with a lens (diopter +1 ) attached to it. This arrangement reduced the impression of a flat screen and eliminated the need for a chinrest. To allow the subjects to produce orientations in depth, a response board was fastened within comfortable operating distance from the subject. The response board was a thin flat square piece of metal with a side length of $18 \mathrm{~cm}$. The visible side of the board was covered with cross-ruled paper. The board was attached to a horizontal axis, and this axis was mounted to a turnable verti. cal shaft so that both rotation axes crossed the middle of the board. The board and part of the axes were placed in a rectangular opening ( $40 \mathrm{~cm}$ long, $20 \mathrm{~cm}$ high), cut out from a vertical screen that served as a reference, representing the $X Y$ plane. To set the board, the subject had to remove his or her eye from the aperture and turn $90^{\circ}$ to the left. In this position, the board was straight ahead of the subject at a distance of approximately $30 \mathrm{~cm}$ and at eye height. The subject could easily handle the board by placing his or her hand on it to turn it to the desired attitude. A simple aiming device made sure that the subject's gaze was perpendicular to the board.

Experimental variation of stimuli. The stimuli were outlined triangles, as shown in Figure 1. They oscillated about a vertical axis. Two main independent variables were used, type of projection and richness of information. Two types of projection were used, parallel and polar. The information variable was also varied in two levels. In the minimal condition, 6 dots were added to the triangle, and in the rich condition, 998 dots were added. A factorial combination of projection type and information richness comprised four main conditions.

To control some proximal parameters, two additional variations were introduced. First, the relation between 2-D and 3-D lengths had to be considered. Since the subjects had to identify the longer of the two lower sides of the simulated 3-D triangle, it was important that relative 3-D lengths of these sides be uncorrelated 

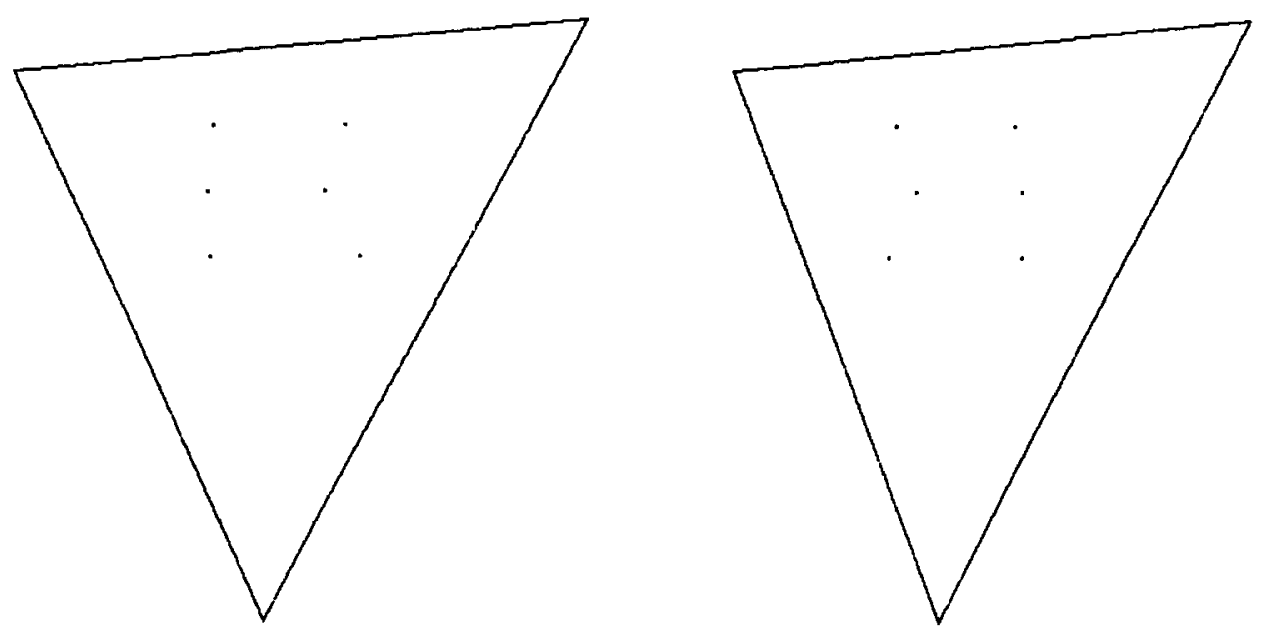

Figure 1. First and eighth frames of a polar projected triangle with six added dots used in Experiment 1.

with 2-D lengths. Therefore, two versions of each triangle were constructed. In one version, the longest 3-D side was also longest in the 2-D projection (A pattern, 2-3-D accordance). In the other version, the longest $3-\mathrm{D}$ side was the shortest side in the $2-\mathrm{D}$ projection (C pattern, 2-3-D conflict). The longest 2-D side also had the swiftest 2-D motion. The use of these two versions made it possible to decide whether the subjects' percepts were mediated by 3-D structure or if 2-D characteristics were used directly. In the case of direct 2-D determination of the percept, it would, of course, not be possible to decide which 2-D factor, length or speed, was the more important. Second, all triangles had a leftright mirrored version in which the spatial layout and motions were mirrored in the vertical dimension.

This means that a total of 16 ( 2 projection types $\times 2$ levels of information richness $\times 2$ types of $2-3$-D compatibility $\times 2$ mirrored versions) different triangles were used. To control for possible carryover effects, a randomized group design was applied with the four unique combinations of type of projection and level of information richness as the four main independent groups. That means that each subject was presented with only one type of projection and one level of information richness, whereas all subjects were shown both types of 2-3-D compatibility and both mirrored versions.

Stimulus parameters. As noted above, the stimulus patterns consisted of simulated triangles rather than only two lines as used by Todd and Bressan (1990). The reason for using triangles was to make it easier for the subjects to form an impression of a surface with a distinct attitude. There might be a tendency to perceive triangles as equilateral that would actually counteract the recovery of perceived euclidean space. However, if information from motion sufficiently well evokes euclidean space, the equilateral tendency should be surpassed by the motion information.

Several important characteristics of the 2- and 3-D triangles cannot, for obvious reasons, simultaneously be kept constant. In the following, compromises between 2-D shape, 3-D orientation, and relative 3 -D lengths of the two sides will be considered.

Two-dimensional shape might affect perceived slant. There is, for instance, a tendency to perceive an equilateral 2-D triangle as oriented closely to the $X Y$ plane. Therefore, the 2-D shapes were made as similar as possible across all triangles. At the start of the rotation, all the 2-D triangles under parallel projection had the same image, the three sides being $13.2,13.7$, and $15.5 \mathrm{~cm}$, starting with the top side and reading counterclockwise. Corresponding values for the polar projection triangles were $12.8,12.8$, and $15.5 \mathrm{~cm}$ for the A patterns and $12.4,12.3$, and $15.5 \mathrm{~cm}$ for the $C$ patterns.
Because the 2-D layout was made similar for the different triangles, it was not possible at the same time to have identical 3-D orientations for the $\mathrm{A}$ and $\mathrm{C}$ patterns which would have been desirable. Extremely large deviations from the $X Y$ plane are seldom perceived from 2-D triangles with about the same extensions in the $X$ and $Y$ dimensions. The attitude in the initial position is described in terms of the direction of greatest deviation from the $X Y$ plane (that is, tilt, $\phi$ ) and in terms of the angular deviation from the $X Y$ plane in the tilt direction (that is, slant, $\theta$ ). In the A patterns, the triangles had $\phi=9.5^{\circ}$ and $\theta=29.4^{\circ}$. Corresponding values for the $C$ patterns were $\phi=19.0^{\circ}$ and $\theta=48.5^{\circ}$.

The two lower sides of a 3-D triangle had a specific difference in length that was supposed to be detected by the subjects. This difference should surpass a minimum size in order to enhance detection and should also be equal for all triangles. However, the introduction of $\mathrm{A}$ and $\mathrm{C}$ patterns and the requirement of similar tilts and slants made it impossible to keep a constant and great difference between the two sides across patterns. In fact, the relative length difference between the lower sides were 1.3:1 for the A patterns and 1.1:1 for the $C$ patterns. A substantial increase in this length ratio for the $\mathrm{C}$ pattern would have resulted in a concomitant unacceptable increase in slant.

Two levels of information richness were accomplished by varying the number of dots added to the triangle. In the minimal condition, 6 dots were added, as shown in Figure 1, forming a rigid 3-D constellation with the triangle. Thus, the minimal patterns contained nine rigidly connected dots. Nine dots were used since 9 is the minimal number of dots necessary to recover a rigid 3-D constellation from two views under polar projection using only linear methods (Longuet-Higgins, 1981). The additional 6 dots were grouped in two subgroups. Each subgroup formed a small vertical triangle that crossed the original big triangle perpendicularly. The small triangles were placed in the upper half of the original triangle, with two of its corners (upper and lower dots) in front of the original triangle and the third corner (middle dot) behind it relative to the observer. The simulated horizontal distance between any of the 6 dots and the original triangle was approximately $10 \%$ of the height of the original triangle. The 6 dots were placed such that they appeared to the authors to be rigidly connected to the triangle under motion. It was not recorded in the experiment if the subjects had the same impression of rigidity. In the rich condition, 998 dots were added. This condition was introduced in order to optimize the conditions for euclidean perception. The dots were placed on the 3-D triangle and on an unbounded plane that slanted $40^{\circ}$ relative to the $X Z$ plane. The 
triangle was transparent, which means that its projected area on the screen had double dot density, with dots derived from both the triangle and the background plane. Regardless of this, the dots were distributed randomly on the screen in the first frame. As was the case in the minimal condition, all additional dots formed a rigid constellation with the 3 -D triangle under rotation. The two information levels actually differed in at least four different respects. First, of course, there was a difference in number of dots. Second, although the triangle was the same, the added dot plane gave the whole pattern a greater visual angle. Third, in the rich condition, there were two surfaces, that of the triangle and that of the background. And fourth, there was a border between these surfaces. These four variables were not separated in this experiment, thus any possible effects of richness could be attributed only to the combined effect of these four variables.

Each simulated 3-D triangle rotated $5.6^{\circ}$ about the $Y$-axis. The apparent rotation was accomplished by showing eight successive views with a stimulus onset asynchrony (SOA) of $83 \mathrm{msec}$ and an interstimulus interval (ISI) of $0 \mathrm{msec}$, which corresponds to values reported by Todd, Akerstrom, Reichel, and Hayes (1988) as optimal under similar conditions. Although, from a theoretical point of view, two views would have been sufficient to provide the 3-D information of polar projections, eight views were chosen to optimize the viewing conditions with smoother motions. Using only two views would have resulted in displacements that would be too great in the $X$ dimension with an angle of rotation of $5.6^{\circ}$. The 3-D triangle rotated $.8^{\circ}$ for each view. The eight frames were run back and forth so that the triangle could oscillate for an appropriate length of time. The choice of $5.6^{\circ}$ was a compromise. On the one hand, it was desirable for the rotations to be as large as possible in order to increase the displacements in the $Y$ dimension, the dimension associated with polar projection. On the other hand, the equipment could not handle more than eight frames unless time or dot-density conditions were changed.

Subjects. Forty naive subjects participated in the experiment. They were undergraduate students of psychology and were not familiar with the problem. Participation was a course requirement. Because carryover effects between the minimal and rich conditions and between polar- and parallel-projection conditions could not be excluded, a between-groups design was used with these variables. Therefore, the 40 subjects were randomly distributed over the four main conditions of the experiment, yielding 10 different subjects in each condition.

Procedure. Each subject had two different tasks. One was to make length judgments, that is, to indicate the longest side of the two lower sides of the triangle. The other was to produce, by means of the response board, perceived orientation in depth. The subjects were requested to produce the orientation at the turning position at which the triangle had the smallest deviation from the $X Y$ plane. Each task was accomplished in a separate session. The order of sessions, length judgments first or productions of orientation first, was counterbalanced between subjects in each group of subjects. Four different stimulus patterns were shown to each subject, that is, the factorial combination of $A$ and $C$ patterns and two versions of the mirrored patterns. Within each session, these four patterns were presented four times, making for a total of 16 presentations for each subject and session. The order of these presentations was randomized so that each subject for each session had a unique order of 16 presentations. Before the length-judgment session, a subject was instructed to decide which of the two lower sides, left or right, had the greatest length by saying "right" or "left" (in Swedish) or by pointing to the right or the left. The subject was given unlimited inspection time in which to make his/her decision. After each response, the next pattern appeared on the screen. Before the orientation-production session, the subject was asked to reproduce the perceived orientation by setting the orientation of the response board. The subject was further instructed about which orientation, the one closest to the $X Y$ plane, to reproduce and how to handle the response board. The subject was allowed to move back and forth between the aperture and the response board until he/she was satisfied with the board setting. The experimenter then recorded the setting, positioned the board to the vertical position, and presented the next pattern. A dim light in the room allowed the subject to operate the board. The tunnel attached to the computer screen assured that the triangles seemed to appear in total darkness.

\section{Results and Discussion}

There was no significant effect of session order, type of projection (polar $=58 \%$, parallel $=63 \%$ ), or interaction between type of projection and information richness on correct length judgments. There was, however, a significant effect of information richness [rich $=66 \%$, minimal $=55 \% ; F(1,36)=8.98, p<.01]$. In summary of the results of length judgments, we have not shown that polar projection as used in the present conditions enhances subjects' ability to recover euclidean structure.

As mentioned above, the attitude of the triangles was expressed in terms of tilt and slant. Several analyses are possible, but we chose to analyze produced slant, that is, the triangle's deviation from the $X Y$ plane. The experimental variation in slant was offered by the difference in slant between the $A$ and $C$ patterns $\left(29.4^{\circ}\right.$ and $48.5^{\circ}$, respectively). An analysis of variance was performed with projection type and information richness as betweengroups variables and simulated slant as a within-group variable. There was a significant main effect of information richness $\left[\right.$ rich $=38.2^{\circ}$, minimal $=32.2^{\circ} ; F(1,36)=$ $5.21, p<.05]$ and of simulated slant $\left[\mathrm{A}=32.6^{\circ}, \mathrm{C}=\right.$ $\left.37.7^{\circ} ; F(1,36)=72.8, p<.0001\right]$. Finally, there was a significant interaction between information richness and simulated slant $[F(1,36)=5.48, p<.05]$, showing that there was a greater effect of simulated slant in the rich condition $\left(\mathrm{C}-\mathrm{A}=6.2^{\circ}\right)$ than in the minimal condition $\left(\mathrm{C}-\mathrm{A}=3.7^{\circ}\right)$. More important is that there were no effects of type of projection.

A related question concerns whether the subjects could at all exploit the extra information provided by polar projection. This might very well be the case even if euclidean percepts, as measured by means of absolute settings, did not show any effect of type of projection. To study this issue we calculated the product moment correlation between simulated slant and produced slant for each subject. For each level in simulated slant ( $A$ and $C$ patterns), each subject made eight settings (two mirrored versions and four replications), all of which were included in the correlation calculations. The correlation coefficients were transformed to Fisher's $Z$ values (Hays, 1973). The mean for each main condition is shown in

Table 1

Group Means of Fisher $\boldsymbol{Z}$ Values in Experiment 1

\begin{tabular}{lcc}
\hline & Parallel Projection & Polar Projection \\
\hline Minimal condition & 0.38 & 0.39 \\
Rich condition & 0.45 & 0.71 \\
\hline
\end{tabular}


Table 1 . There is a significant difference in $Z$ values between polar and parallel projection in the rich information condition $[t(18)=-2.31, p<.05]$. The combination of polar projection and rich information makes the slant settings more consistent and dependent on simulated slant. For instance, in this condition, $37.3 \%$ of the variance is accounted for by experimental variation. Corresponding values are $17.8 \%$ for parallel/rich, $13.8 \%$ for polar $/ \mathrm{minimal}$, and $13.1 \%$ for parallel $/ \mathrm{minimal}$. Although euclidean space perception has not been demonstrated, the data show that polar-projection information in the rich information condition makes slant judgments more consistent and dependent on simulated slant.

The second aim of Experiment 1 concerned the question of whether the length judgments could be accounted for by an internal mechanism that randomly assigned values to some rotation-related measure. Todd and Bressan (1990) have pointed out that their obtained percentages of correct length judgments (for stimuli very similar to ours) could be accounted for if the estimated angles of rotation $(\varepsilon)$ were evenly distributed over $1 / \varepsilon$, ranging from about 5 to 80 radians $^{-1}$ and corresponding to an angle of rotation ranging from just above $10^{\circ}$ to a little less than $1^{\circ}$. They had no data on perceived angle of rotation. Although we did not directly measure the perceived angle of rotation, it was possible to estimate the value of $\varepsilon$ used by the subjects in the slant-production

\section{Polar projection - minimal condition}

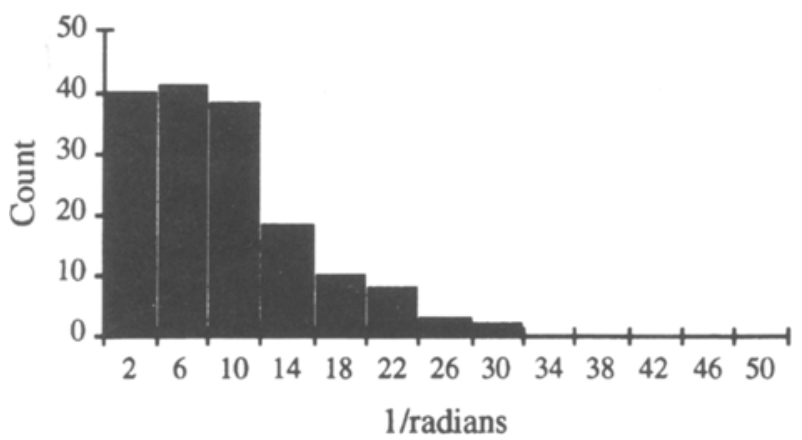

Parallel projection - minimal condition

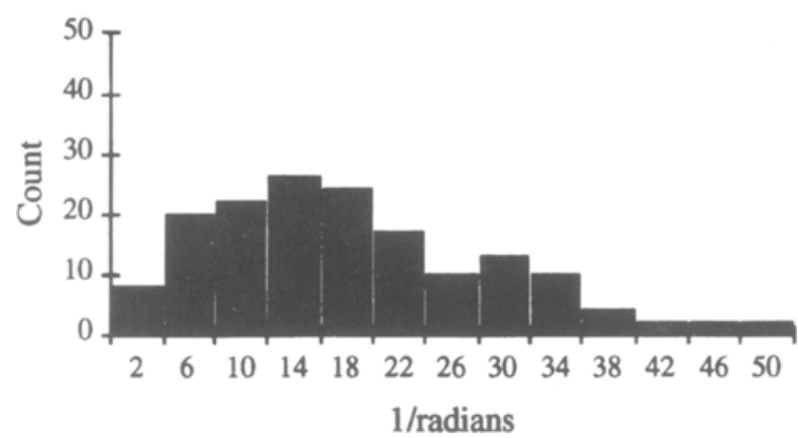

task on a trial-by-trial basis. Todd and Bressan (1990) showed that, for small displacements, the proximal velocity of a point is the product of the angular displacement of the distal point in radians and the $Z$ distance of the point, given that the point is rotating about an axis parallel to the picture plane and that parallel projection is used (see Equation 3 below). The produced slants in our experiment indicate the subjects' perceived $Z$ distances. Following Todd and Bressan (1990), that is, considering the mathematical relationship between proximal velocity, angular displacement, and $Z$ distance, to be relevant to human perception, the obtained $Z$ distances (by means of produced slants) can be used to calculate perceived angular displacement. To minimize estimation errors, the fastest moving point in each triangle was used in these calculations. Figure 2 presents these data transformed to $1 / \varepsilon$, as expressed in radians. As can be seen, the distributions are not rectangular, and almost all judgments fall below 30 radians $^{-1}$. Furthermore, the judgments do not seem to be stimulus independent. At least for the rich conditions, the median is close to the theoretical value of 10.23 . To conclude, our data do not show a random assignment of $1 / \varepsilon$ that results in a rectangular distribution. One reason for that might of course be that there is no such assignment. Another reason might be that the mathematical relationships referred to do not hold for perceptual processes.

\section{Polar projection - rich condition}

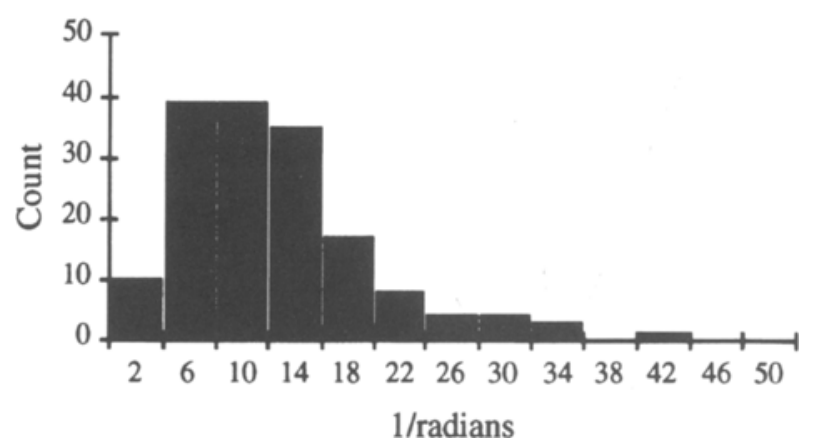

Parallel projection - rich condition

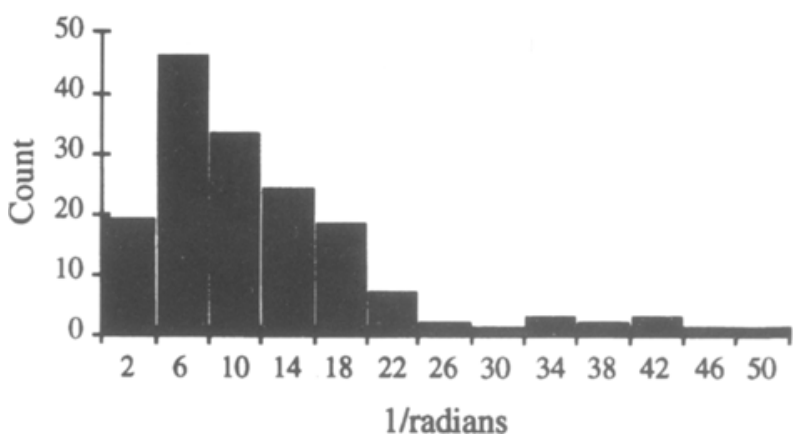

Figure 2. Distribution of calculated perceived rotational displacements in Experiment $1(N=160,10$ subjects $\times 16$ judgments $)$. 


\section{EXPERIMENT 2}

Experiment 2 was a renewed attempt to demonstrate euclidean structure from polar-projected motion by using greater visual angles and smooth surfaces.

Euclidean structure from motion was not demonstrated in Experiment 1. However, it still might be that information in polar projection contributes to the recovery of Euclidean structure, although the results from Experiment 1 did not show that. One obvious way to increase the possible effects of polar projection is to increase the visual angle. Another way, suggested by Experiment 1 , is to add more dots and surfaces since these have been shown to affect the number of correct length judgments. The importance of continuous-flow fields extended over an area have been stressed by Lappin (1990). Therefore, larger visual angles and smooth surfaces were used in Experiment 2. This was accomplished by showing a dot pattern simulating a sinusoidal protuberance directed toward the subject and rotating about a vertical axis. The stimulus patterns covered visual angles of $4.35^{\circ}$ or $17.40^{\circ}$, and the subjects had to produce the perceived height:width ratio of the protuberance on a response monitor. Experiment 2 had two objectives. The first, and more ambitious one, was to demonstrate, if possible, euclidean recovery of structure from motion, at least at great visual angles. The second objective was derived from results in Experiment 1 which had shown an increased covariation between simulated and produced slant (in the rich condition) when polar projection was substituted for parallel projection. It was hypothesized that, at the greater visual angle, there should be a greater covariation between simulated and produced height: width ratios. This does not necessarily mean that the percepts become euclidean - the scale factor in depth might still be different from the one in the $X Y$ plane - but it implies that the information about simulated depth is used more consistently and reliably.

\section{Method}

Apparatus and viewing conditions. The stimulus patterns were displayed by a monochrome 21 -in. Super Mac monitor, Type MM2136ASM, with a display area of $39.2 \times 28.5 \mathrm{~cm}$ and a resolution of $77 \mathrm{dpi}$. The distance between the subjects and the display monitor was either 379 or $94 \mathrm{~cm}$. A response keyboard and monitor were used by the subjects to reproduce perceived height:width ratios. The response monitor was a monochrome Apple SE monitor with a display area of $17.9 \times 11.6 \mathrm{~cm}$ and a resolution of $72 \mathrm{dpi}$. It was placed about $45^{\circ}$ to the left, relative to the direction to the stimulus display monitor, and at a distance of $50 \mathrm{~cm}$ from the subject. The keyboard was placed about $20 \mathrm{~cm}$ in front of the subject at a comfortable operating distance. As shown in Figure 3, the response monitor displayed two vertical lines of 330 and 224 pixels. The left line had two marks on it separated by 224 pixels and corresponding to a visual angle of $9^{\circ}$. The subject could move the right line horizontally by pressing appropriate keys in small, 1-pixel, or large, 5-pixel, steps.

Stimuli. Since Experiment 1 had shown effects of the number of dots, a great number of dots were used in the stimulus patterns in order to enhance the possibility of reconstructing euclidean structures. To further increase information, smooth surfaces were

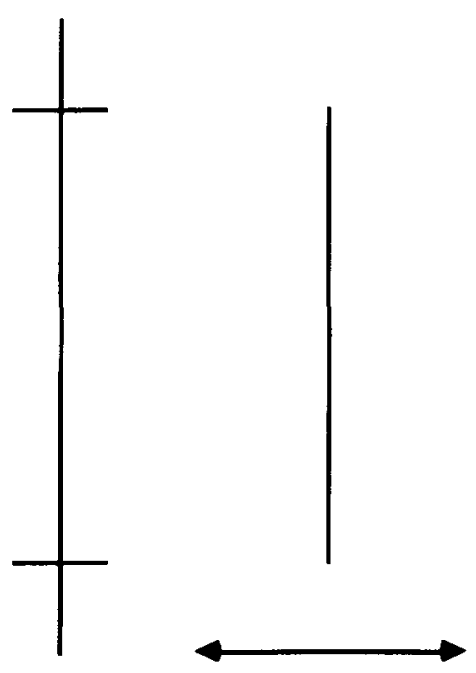

Figure 3. Response figure in Experiments 2 and 3. The right vertical bar could be moved by the subject, by means of a keyboard, to indicate the perceived height:width ratio.

simulated (e.g., Lappin, 1990; Todd \& Norman, 1991). Each stimulus pattern consisted of approximately 8,000 dots simulating a (co)sinusoidally shaped concentric protuberance with its peak along the $Z$-axis, pointing at the subject. The protuberance represented a complete period, which means that the height of the protuberance equaled the doubled amplitude. At the base, there was a smooth transition to a plane, still simulated by dots, that continued beyond imaginary borders that framed a square with a side of 870 pixels. It was important to strengthen the impression that the dot surface really was situated beyond the imaginary borders so that the shape of the imaginary square opening did not affect the perception of the whole dot constellation. Therefore, we were careful to let the dots close to the borders appear and disappear between frames according to the polar projection of the simulated surface through the imaginary square opening. The plane was perpendicular to the protuberance and rigidly connected to it. When the protuberance pointed straight toward the subject, all dots were randomly distributed in the $X Y$ plane so that no 2-D cues from a single frame were provided. The apparent rotation in depth about a $Y$-axis through the center of the protuberance was accomplished by showing two successive views. These views were alternated (SOA of $200 \mathrm{msec}$ and ISI of $0 \mathrm{msec}$ ), giving the protuberance an oscillating motion. Three main variables were varied in the experiment. Visual angle was varied at two levels, $17.40^{\circ}$ and $4.35^{\circ}$. These angles describe the width of the protuberance at its bottom. This variation was brought about by changing the images to simulate polar projections of $17.40^{\circ}$ and $4.35^{\circ}$ and changing the distance between the display screen and the subject accordingly. As a consequence, there was a change of dot density and resolution, the latter being 200 pixels per degree in both $X$ and $Y$ dimensions at the greater distance and 50 pixels per degree at the smaller distance. The visual angle of each dot was, however, kept constant by changing its $2-\mathrm{D}$ size $(4 \times 4$ pixels at the greater distance and 1 pixel at the shorter distance). The simulated height of the protuberance was varied in two levels, $1.0: 1$ and $0.7: 1$ relative to the base. Finally, the rotational displacement about the $Y$-axis was varied. The displacements used were $1.5^{\circ}$ or $3.0^{\circ}$. Since the exposure-time conditions were the same for all patterns, there was a concomitant change in rotation speed.

Subjects. Eight subjects participated in the experiment. They were undergraduate students of psychology and were not familiar with the problem. Participation was a course requirement. 
Procedure. Before the experiment, the subjects were shown a pattern similar to those shown in the experiment. They were instructed on how to operate the keyboard. The task was to judge the height of the protuberance relative to its width at the base, and to reproduce this height:width relationshp by setting the right vertical line on the response monitor. The lines on the monitor represented a side view of the measures of the protuberance. The distance between the two marks on the left line represented the width of the protuberance at its base, and the distance between the two vertical lines represented the height of the protuberance. When the subjects had been shown how to move the right line on the response screen by using the keyboard, the experiment was begun. All subjects were presented with all patterns. First, all patterns at one distance were shown, and then all patterns at the other distance were shown. Order of distance was counterbalanced over subjects. At each distance, there were four different stimulus patterns (two height:width relations $x$ two angles of rotation). These were randomized into three blocks, with each pattern appearing once in each block. The two first blocks were run just to familiarize the subjects with the task; only data from the third block were analyzed. In this way, one measurement per condition was obtained from each subject, giving a total of eight observations for each subject. The subjects were given unlimited inspection time in which to make their decisions. After each judgment, the subject pressed a key for the next pattern to be shown. A dim light in the room allowed the subject to operate the keyboard.

\section{Results and Discussion}

There was one significant main effect, visual angle $[F(1,7)=30: 95, p<.01]$ and one significant interaction, visual angle $\times$ simulated depth $[F(1,7)=6.09, p<.05]$. Figure 4 shows this interaction. It is clear from the figure that visual angle has an effect on perceived structure. At the small visual angle, the subjects could not discriminate simulated height:width ratio at all, whereas at the larger angle they could. Figure 4 also shows that the produced ratios did not match the simulated ratios. Even at large visual angles, the greater simulated height:width ratio (1.0) is underestimated. Our conclusions from these

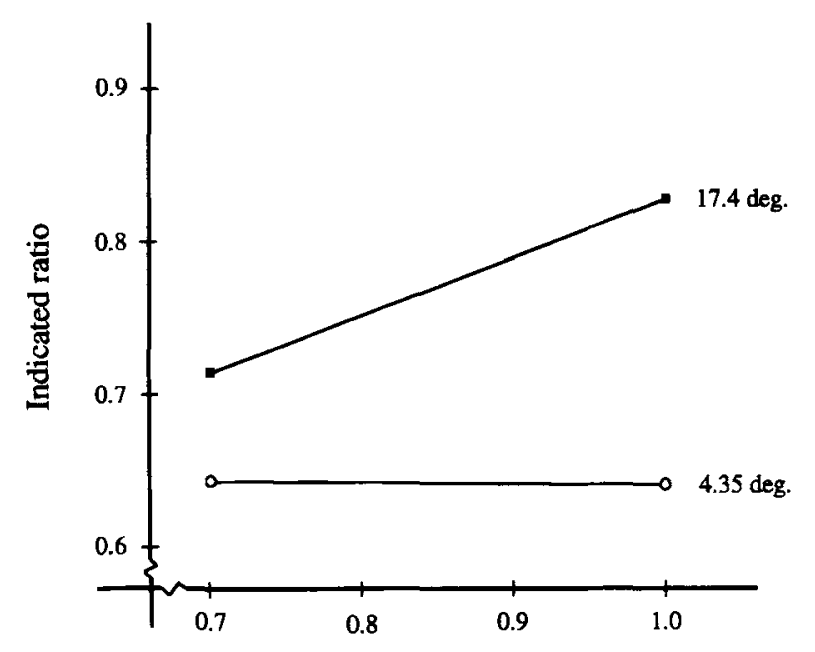

Simulated ratio

Figure 4. Produced height:width ratios in Experiment 2. The interaction indicates that polar projection information is used to discriminate depth only at the larger visual angle $\left(17.4^{\circ}\right)$.

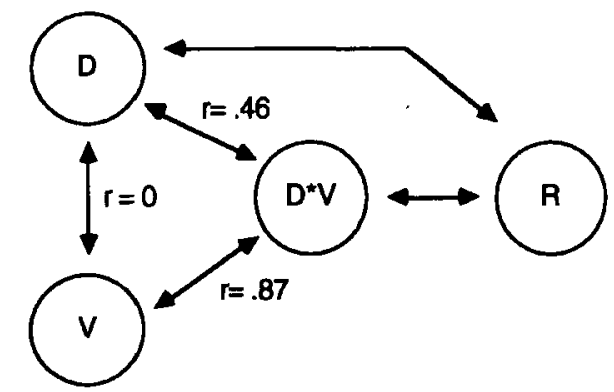

Figure 5. Correlations between the 2- and 3-D variables in Experiment 2. $D$, simulated height:width ratio; $V$, simulated angle of rotation; $D * V$, the proximal velocity of the dots on the screen (for an explanation, see text).

results are, first, that euclidean recovery of structure was not shown, and second, that information about simulated height:width ratio is more consistently used at larger visual angles. Whether this increase in consistency is dependent on polar projection information as such or on the greater visual angle, irrespective of projection type, cannot be determined by the present experiment. Experiment 4 below will shed some light on this issue.

An important aspect of the results concerns the effect of rotational displacement. In parallel projection, it is not possible, with the present kind of patterns, to determine to what degree the changes in two successive images are brought about by height:width ratio, on the one hand, and angle of rotation, on the other. We found a nonsignificant effect of angle of rotation. With an increase of angle (from 1.5 to 3.0), there was an increase in the means of height:width ratio judgments (from .67 to .75 ). It should be noted that the variation of rotational displacement used in this experiment leads to approximately a doubling of the displacements of the dots from one view to another (at the center top of the protuberance), whereas an increase in simulated height:width ratio from $0.7: 1$ to 1.0:1 leads only to about a $40 \%$ increase. In spite of that, the variation of height:width ratio had greater effects on perceived structure than did variation of rotational displacement.

One way to evaluate perceptual processes is to match absolute values of judgments with absolute values of simulated or distal stimuli. By doing this, it was shown that euclidean structure was not recovered in this experiment. However, there was a covariation between simulated and perceived height:width ratio with large visual angles. The origin of this covariation was further analyzed by means of correlations that excluded the scale factor and stressed the relative contribution of different information sources. In particular, the analysis was undertaken to see whether an affine analysis could account for the results. In Figure 5, the four relevant variables are shown as circles: $V$ is the simulated angle of rotation $\left(1.5^{\circ}\right.$ and $\left.3.0^{\circ}\right), D$ is the simulated height:width ratio $(0.7$ and 1.0 ), and $D * V$ is the proximal velocity of the dots on the screen. The $r$ s between the circles denote the product moment correlations between these variables and are, of 
course, determined by the choice of stimulus patterns. The fourth circle, $R$, represents the subjects' responses. For each subject, it was possible to calculate a correlation between $R$ and any of the other three variables on the basis of the subject's responses. Only judgments from the large-visual-angle condition were used, since no effects of simulated height:width ratio were found in the small-visual-angle condition. The two correlations between $R$ and $D$ and between $R$ and $D * V$ are both means of individual correlations. These means were calculated after appropriate Fisher $Z$ transformations were made (Hays, 1973). Admittedly, the situation is not optimal for correlation analysis because the three stimulus variables are fixed variables and the coefficients for correlations between $R$ and the stimulus variables are based on only four data pairs. Nevertheless, we think that the analysis reveals interesting information. The question concerns the kind of information on which the visual processes are based. An affine analysis says that $r(D \times V, R)$ for each subject would equal 1.0. As a consequence the correlation for each subject between $D$ and $R, r(D, R)$ would equal $1.0 \times .46=.46$. Euclidean processing, on the other hand, says that $R$ is entirely dependent on simulated height:width ratio, $r(D, R)=1$. A statistical test was performed to see whether an affine solution was compatible with the data. For each subject the difference, $r(D, R)-$ $r[(D \times V), R) \times r[(D \times V), D]$, was calculated after appropriate Fisher $Z$ transformations were made. A positive difference suggests that the subjects utilized information from simulated height:width ratios to a greater extent than can be explained by complete affine processes. The mean of the difference from the 8 subjects was $.37[t(7)=2.44$, $p<.05]$. Furthermore, the simulated height:width ratio accounts for $38 \%$ of the variance, whereas an affine explanation, taking into account the obtained $r[(D \times V), R]$, would predict that simulated height:width ratio should account for $11 \%$ of the variance. This analysis of correlations clearly shows that there is something more to the perception of height:width ratios than an affine analysis.

This type of analysis cannot replace analyses of absolute performances but can reveal patterns of processing that do not necessarily show up when such performances are scrutinized.

\section{EXPERIMENT 3}

Experiment 2 showed that the information added by polar projection at somewhat larger visual angles facilitated consistent recovery of structure (although not euclidean) from motion. The increase in visual angle leads to a concomitant increase in deviation from the horizontal direction of the 2-D motion paths, which was taken to be the crucial variation. In Experiment 2, however, dot density was allowed to covary with visual angle. Experiment 3 was run as a control experiment to rule out the possibility that dot density could account for the interaction between visual angle and simulated height:width ratio demonstrated in Experiment 2.

\section{Method}

Apparatus and viewing conditions. The apparatus and viewing conditions were the same as those in Experiment 2 except that only one viewing distance, $379 \mathrm{~cm}$, was used.

Stimuli. The stimuli were similar to those used in Experiment 2 . The same protuberance was simulated under the same time parameters and number of views. Only one visual angle was used $\left(4.35^{\circ}\right)$. However, the density of the dots was varied in three steps. At the highest density, 8,000 dots, the pattern was identical to that shown at $4.35^{\circ}$ in Experiment 2. The middle level of density included 1,952 dots; the least density had 312 dots. The least dot density corresponded approximately to the dot density in the patterns shown at $17.4^{\circ}$ in Experiment 2. Two 3-D height:width ratios were used, $.7: 1$ and 1.0:1, and two angles of rotation in depth were used, $1.5^{\circ}$ and $3.0^{\circ}$. This resulted in the 12 different patterns used in Experiment 3.

Subjects. Eight subjects participated in the experiment. They were undergraduate students of psychology and were not familiar with the problem. Participation was a course requirement.

Procedure. The subjects were shown a pattern similar to those to be shown in the experiment and were instructed in how to operate the keyboard. Then three blocks of patterns were presented to the subjects. Each pattern appeared once in each block. The order of the patterns was randomized within each block. The first two blocks were given to familiarize the subjects with the task. Only data from the last block were analyzed. The subjects were given unlimited inspection time in which to make their decisions.

\section{Results}

An analysis of variance revealed that there was a significant effect of dot density $[F(2,14)=4.93, p<.05]$. The difference appeared between the condition with the highest dot density $(8,000$ dots $=.67)$ and the other two conditions $(1,952$ dots and 312 dots $=.77)$. There was also a significant effect of angle of rotation $[F(1,7)=$ $12.86, p<.01]$. The produced mean in height:width ratio was .68 for $1.5^{\circ}$ of rotation and .79 for $3.0^{\circ}$ of rotation. This difference indicates poor performance. The subjects could not differentiate information about internal depth and information about rotation in depth. No other sources of variance were significant, not even simulated height:width ratio, showing that the subjects were poor at reproducing height:width ratios. It is obvious that polar projection at a visual angle of $4.35^{\circ}$ under these conditions provides inadequate information for the requested task. It is of special importance to note that there was no interaction between simulated height:width ratio and dot density. Experiment 2 showed an interaction between simulated height:width ratio and size of visual angle. Consequently, this interaction cannot be interpreted in terms of simulated height:width ratio and dot density.

\section{EXPERIMENT 4}

Experiment 2 had shown that although the recovered perceived space was not euclidean, performance was better than could be accounted for by a strict affine analysis. The present experiment dealt further with this question. In particular, in Experiment 4, three possible information sources and corresponding analyses for recovery of depth from motion were scrutinized using only two frames. The first source comprised two independent mea- 
sures, one being dot velocities in the $X$ dimension and the other being dot velocities in the $Y$ dimension. These two measures are required for a euclidean analysis (but of course do not guarantee such an analysis). The second source consisted of one measure, that is, dot velocities in the $X$ dimension. An analysis of $X$ motions only is sufficient for an affine analysis but not for a euclidean analysis. Finally, the third source consisted of one measure that was a combination of dot velocities in both $X$ and $Y$ dimensions. Similarly, this analysis can recover affine but not euclidean structure. By experimentally manipulating the $X$ and $Y$ changes (rather than the simulated objects), it was shown that the perceived shapes deviated significantly from the predicted affine solutions. On the contrary, perceived shapes were consistently related to simulated shape according to a euclidean analysis. This implies that the visual system utilizes the dot velocities in the $X$ and $Y$ dimensions independently to recover structure. However, the slopes relating perceived and euclidean depth/width ratios were substantially smaller than 1 .

The projected motions on the $X-Y$ plane of the individual dots on a 3-D object rotating about the $Y$-axis, were analyzed in the $X$ and $Y$ dimensions separately. With the projection point placed at $z=1$, the $Y$ motion of an arbitrary dot can be expressed as

$$
\dot{y}=x y \dot{\alpha},
$$

where $\dot{y}$ denotes the projected $Y$ velocity, $x$ the projected $X$ position, $y$ the projected $Y$ position, and $\dot{\alpha}$ the 3-D rotational velocity of the dot. The $X$ motion of an arbitrary dot on the 3-D object is described by Equation 2,

$$
\dot{x}=x^{2} \dot{\alpha}-D \dot{\alpha},
$$

where $\dot{x}$ denotes the projected $X$ velocity and $D=z /(1-$ $z$ ). As can be seen, $\dot{x}$ is determined by $\dot{\alpha}$ and $z$ jointly.

To obtain a euclidean structure from two views only, the two measures $\dot{y}$ and $\dot{x}$ are needed, as shown above. An affine solution needs only one measure for each dot. There might be several candidates for such a measure. Two possible measures were considered. One possible measure would be $\dot{x}$ according to Equation 2. A system that works according to this principle was called a parallel affine system, since motions only in the $X$ dimension are registered. The other affine system considered used motions in the $Y$ dimension as well. Using parallel projections, Todd and Bressan (1990) have shown that $\dot{x}$ is dependent on rotational displacement and depth only, irrespective of visual angle $(x)$ which, with our notation, can be expressed as

$$
\dot{x}=-\dot{\alpha} z .
$$

Since Todd and Bressan (1990) used this formula when discussing the characteristics of the visual system, it would be interesting to study a measure in terms of $X$ and $Y$ motions that corresponded to $\dot{\alpha} z$. From Equations 1 and 2, it follows that

$$
x\left(\frac{\dot{y}}{y}-\frac{\dot{x}}{x}\right)=\dot{\alpha} D .
$$

Since $D$ approximates $z$ for small values of $z, x[(\dot{y} / y)-$ $(\dot{x} / x)]$ was taken as an acceptable approximation for $-\dot{\alpha} z$, which can be applied for large visual angles (see Lind, in press). A system that analyzes $x[(\dot{y} / y)-(\dot{x} / x)]$ was called a polar affine system since both $X$ and $Y$ motions were taken into account when calculating a measure for affine analysis.

To summarize, the purpose of Experiment 4 was to compare a euclidean analysis based on two independent measures, $\dot{x}$ and $\dot{y}$, with two affine analyses based on different measures; a parallel affine analysis based on $\dot{x}$ and a polar affine analysis based on $x[(\dot{y} / y)-(\dot{x} / x)]$. This was accomplished by independently and systematically varying 2-D motions in the $X$ and $Y$ dimensions.

\section{Method}

Stimuli. All patterns consisted of two alternating frames (SOA of $200 \mathrm{msec}$ and ISI of $0 \mathrm{msec}$ ) with approximately 6,000 dots. Since the displacement of a dot could not be a fraction of a pixel, an anti-aliasing technique was introduced to enhance the impression of smooth movements. Each dot was defined by an area of $3 \times 3$ pixels. Between four and nine of these pixels were always illuminated by one of 15 intensity levels related to the distance between the theoretical position of the dot and the position of a specific pixel. Informal observations before the experiment had shown that the motions of the dots appeared much smoother and that the impression of the dots remained distinct with this technique. Each frame covered a visual angle of $25^{\circ} \times 33^{\circ}$ in the $Y$ and $X$ dimensions, respectively. The dots were distributed randomly on the $X Y$ plane on one of the frames. The relatively small displacements of dots between frames did not change the impression of randomness in the other frame. The two frames simulated a 3-D vertical ridge with a sinusoidal profile, with the top directed along the $Z$ axis toward the subject. The frames were covered by 1.4 periods of the sine wave. The ridge rotated about a vertical axis.

The construction of patterns started from a basic pattern with the following properties. The $\Delta y$ value for each dot was assigned according to Equation 1 so that a rotational displacement of $\Delta \alpha=$ .75 was simulated. The $\Delta x$ value for each dot was assigned according to Equation 2, so that a sinusoidal ridge with 1.4 periods and with a height:width ratio of $65: 1$ was simulated. Since a complete period was shown, the height was equal to twice the amplitude.

The $\Delta y$ and $\Delta x$ values were varied in the following ways: The $\Delta y$ values were increased in 12 steps so that 13 two-frame patterns with increasing values of $\dot{\alpha}$ were obtained. The quantity $\Delta \alpha$ varied from $.75^{\circ}$ to approximately $3^{\circ}$. The $\Delta \alpha$ values were chosen so that the simulated height: width ratio decreased in equal steps of .04 from $.65: 1$ to $.17: 1$. This series of $\Delta y$ values was used in all conditions of Experiment 4.

Considering $\Delta x$ values, two series were chosen. In the first series, Condition 1 , the $\Delta x$ values from the basic pattern were kept constant across the 13 patterns with different $\Delta y$ values. The reason for constructing stimuli in this way was to differentiate a euclidean analysis from an affine analysis on the basis of $X$ motions only (parallel affine analysis). If euclidean structure is recovered, there will be a positive correlation between simulated depth and reproduced depth across the 13 patterns with a slope close to 1 . (For a system without errors of measurement, $r=1$ and the slope, $k=1$.) An affine solution based on $\dot{x}$ values only would yield no correlation between simulated depth and produced depth, since the $\Delta x$ values did not vary across patterns. There is a third alternative with a significant correlation but a slope that substantially deviates from 1 . This outcome implies that the visual system, in accordance with a euclidean analysis, independently analyzes $\dot{x}$ and $\dot{y}$ values to obtain perceived depths that are consistently re- 
lated to simulated depths but fails to match the scale factor in the $Z$ dimension with that in the $X$ and $Y$ dimensions.

The second series, Condition 2 , had the same $\Delta y$ values as the first series. However, rather than keeping the $\Delta x$ values of the basic pattern constant across patterns, as was the case in Condition 1, the $\Delta x$ values were varied across the 13 patterns so that $x[(\Delta y / y)-(\Delta x / x)]$ remained constant across patterns. In other words, the $\Delta x$ value of a particular dot in any pattern in Condition $1, \Delta x_{1}$, was substituted for a $\Delta x$ value in Condition $2, \Delta x_{2}$, such that $\Delta x_{1}=x\left[(\Delta y / y)-\left(\Delta x_{2} / x\right)\right]$. The reason for constructing the stimuli in this way was to differentiate a euclidean analysis from an affine analysis based on one measure combining motions in both $X$ and $Y$ dimensions (polar affine analysis). If euclidean structure is recovered, there will be a positive correlation between simulated and produced depth across the 13 patterns with a slope close to 1 . If the visual system makes an affine analysis based on $x[(\dot{y} / y)-(\dot{x} / x)]$, there will be no correlation between simulated depth and produced depth across patterns, since $x[(\Delta y / y)-(\Delta x / x)]$ did not vary. As described for Condition 1 , there is a third alternative according to which the visual system analyzes $\dot{x}$ and $\dot{y}$ values independently but fails to match the scale factor in the $Z$ dimension with that in the $X$ and $Y$ dimensions.

Apparatus and viewing conditions. The apparatus was the same as that used in Experiment 2. The display monitor was placed at a distance of $63 \mathrm{~cm}$ in front of the subject, who looked at the displays monocularly. The viewing position was controlled by a chinrest. The response monitor was placed at a distance of $32 \mathrm{~cm}$ from the subject and about $45^{\circ}$ to the right relative to the direction of the display monitor. The response pattern was initially a straight vertical line of 269 pixels. By pressing appropriate keys on the keyboard, the subject could turn the line into a sinusoidal shape and control its amplitude. The sine wave showed the profile of the simulated ridge as seen from above with the subject's position to the right of the line.

Subjects. Ten naive psychology students and the authors participated as subjects. The students' participation was a course requirement. No differences were found between the students' and the authors' judgments.

Procedure. The subjects were informed about the task and told how to operate the keyboard. It was pointed out to them that they should let their eyes drift over the pattern in order to avoid any adaptation effects that would possibly blur the image. Eight patterns, selected randomly from the experimental patterns, were shown, and the subjects adjusted the response line in order to get acquainted with the task. After a short break, the 26 experimental patterns were shown in randomized order. Each subject received a unique set of training patterns and a unique order of experimental patterns. The subjects were given unlimited inspection time in which to make their decisions. The time interval between a decision and the appearance of the next pattern was about $60 \mathrm{sec}$. During this time, the display screen was dark.

\section{Results}

The correlation between simulated depth and produced depth was calculated for each subject and condition. Each correlation was based on 13 pairs of data. The $r$ s were transformed to Fisher $Z$ scores. The mean of the $Z$ scores for Condition 1 over subjects was .308. This mean was $t$ tested and found to be significantly greater than zero $[t(11)=2.296, p<.025]$. It is concluded that the visual system does not make a parallel affine analysis based on motions in the $X$ dimension alone. The mean of the $Z$ scores for Condition 2 was .480 , which also was significantly greater than zero $[t(11)=5.879, p<.0001]$. It is concluded that the visual system does not make a polar affine analysis based on $x[(\dot{y} / y)-(\dot{x} / x)]$. Although other measures for an affine analysis cannot be decisively rejected, it is concluded that the produced depths were controlled by a process that computes $\dot{x}$ and $\dot{y}$ values independently. This is exactly what a euclidean analysis does. However, the resulting percepts are still not euclidean. For each subject and condition, the slope relating produced and simulated (according to a euclidean analysis) width:depth ratios was calculated. The slopes are substantially smaller than 1 , with a mean of .24. Summarizing the results, it is concluded that the visual system utilizes changes in both $X$ and $Y$ dimensions independently to recover simulated depth, and that the perceived variation in the depth dimension is underestimated.

\section{GENERAL DISCUSSION}

The general aim of this study was to ascertain the ability of the visual system to reconstruct perceived euclidean structure from motion. In spite of reported failures to find such an ability and even statements about the inevitable affine character of the system (Koenderink \& van Doorn, 1991; Todd \& Bressan, 1990; Todd \& Norman, 1991), it was hypothesized that euclidean structure from motion under appropriate conditions could be demonstrated. As noted by Todd and Bressan (1990), their results were limited to parallel projections, and it remained to be determined whether polar projections offered sufficient information for the perception of euclidean structure. We have not been able to demonstrate this kind of perception. In Experiment 1, there was no increase in correct length judgments when polar projection was used. To enhance the possibility for recovery of euclidean structure, Experiment 2 was designed with somewhat larger visual angles and smoothly curved surfaces as stimuli. Experiment 2 showed that the subjects detected the differences in simulated depth under a relatively large visual angle which they did not detect under a small visual angle. We have interpreted this outcome as an effect of the additional information provided by the polar projection. There are, however, other differences between the two visual-angle conditions that might have had an effect. First, there is the target's visual angle, as such, to be judged. One way to control the effect of the target's visual angle would be to keep it constant. We have made some preliminary studies with constant size of target but with different visual angles of a background that has been rigidly connected to the target. The results obtained so far indicate that the correlation between simulated depth and perceived depth increases when the visual angle of the background increases. It seems that the target's visual angle, as such, is not critical inasmuch as reliable polar information can be obtained in some way, for instance, from the background. A second difference between small and large visual angles concerns dot velocities in the image plane. Since simulated shape was kept constant in Experiment 2, there was a difference in absolute simulated depth between small- and largevisual-angle conditions. This affects both absolute and relative velocities. For instance, an arbitrary dot on the 
target has a greater proximal velocity under large visual angle than under small visual angle. The same holds true for the difference in proximal velocity between two arbitrary dots. Although the contribution of these proximal velocities should be studied in greater detail, there is information that suggests that they cannot account for the differences in judged depth for the small- and largevisual-angle conditions obtained in Experiment 2. The interaction between simulated depth and size of visual angle, especially, cannot be explained only by the greater proximal velocity patterns with the larger visual angles. The preliminary study mentioned above also suggests that proximal velocities of the target cannot exclusively determine perceived depth.

Experiment 4 provided further evidence that polar information is used in the analysis by a process in which the changes in the $X$ and $Y$ dimensions are treated independently. It was shown that affine processes utilizing $\dot{x}$ or $x[(\dot{y} / y)-(\dot{x} / x)]$ as measures could not account for the results. One could ask if there were other 2-D measures that could be the basis for an affine analysis resulting in the obtained positive correlations. This seems less likely, since most measures based on velocities would, in fact, be expected to result in a negative correlation between velocity and perceived depth in Experiment 4 . Generally, when the 2-D differences in velocity between dots increase in a motion parallax display, greater distances in depth are simulated and perceived. The stimulus patterns in Experiment 4, however, were constructed the other way around. An increase in $\dot{y}$ values across patterns within each condition in Experiment 4 implied greater velocities of the dots not too close to the $X$-and $Y$-axes (see Equation 1) and no, or very small, changes in the velocity of dots close to these axes. As a consequence, an increase in $\dot{y}$ values across patterns led to greater velocity differences between dots within the patterns. An increase in $\dot{y}$ values also resulted in smaller simulated height:width ratios. A reliance on general size of velocity vectors would therefore result in a negative correlation. However, a positive correlation was obtained, as predicted by a euclidean analysis.

Additional informal support for the utilization of polar information was given in Experiment 4 by spontaneous reports from the subjects indicating perceived differences of rotational displacements, $\dot{\alpha}$. These differences must have been dependent on the $Y$ changes (see Equation 1). Since the determination of $\alpha$ may play a crucial role in the analysis of depth, it would be interesting to study directly the perception of angle of rotation in these types of studies. We are preparing such studies.

Many studies have shown that affine structure can be obtained with great precision by observers, whereas recovery of euclidean space from motion remains to be demonstrated. The addition of polar information in our experiments did not yield euclidean percepts. This does not, however, imply that this information is not used. On the contrary, it has been shown that changes in the $Y$ dimension are systematically used by observers to determine depth. For instance, in Experiment 1, there was a significant correlation between simulated slant and produced slant under polar projection with many dots. Experiments 2 and 4 also showed that dot velocities in the $Y$ dimension were utilized. However, the correlations between simulated and produced depth were substantially lower than 1 . One reason for that might be limited precision in the visual system. Under small visual angles, a very small noise in the measurements of retinal velocities produces great errors. Koenderink and van Doorn (1987) have shown that an error variance at, for instance, $5 \%$ makes euclidean analysis impossible if the visual angle is less than $15^{\circ}$. A perfect recovery of $\dot{\alpha}$, a prerequisite for an analysis of euclidean structure, requires visual angles larger than $100^{\circ}$. In light of this, the obtained correlations of .3-.4 at visual angles of about $30^{\circ}$ suggest that the visual system makes as consistent depth judgments as can be expected, taking errors of measurement into account. High correlations between simulated and perceived depth do not guarantee euclidean space recovery. There should also be a match in absolute magnitude. Our data show slopes that are less than 1 , indicating that the variation in perceived depths is underestimated. This is also a general finding for perceived depth from binocular stereopsis. The horizontal-vertical illusion is another example of a systematic effect that can disguise an otherwise more or less correctly working mechanism. Geometrical illusions can even violate euclidean analyses restricted to the $X Y$ plane. Norman, Todd, Perotti, and Tittle (1996) and Tittle, Todd, Perotti, and Norman (1995) have recently shown, for 3-D lengths and 3-D shapes, respectively, that constancy is not retained when orientation or distance relative to the 3-D object is changed. Furthermore, the task in Experiments 2-4 demanded a mental rotation of shapes that might have effects on the reports. Consequently, there are several factors that might affect the percepts such that euclidean structure does not appear. However, it still seems to be the case that $Y$ changes (together with $X$ changes), within limits of the precision of the visual system, yield consistent depth percepts. A one-to-one mapping between real and perceived depth that is consistent over time and variations in velocity might do quite well in ordinary life, even if the scale factor in the $Z$ dimension does not coincide with the one (or one of two) in the $X Y$ plane.

\section{REFERENCES}

Bennett, B., Hoffman, D., Nicola, J., \& Prakash, C. (1989). Structure from two orthographic views of rigid motion. Journal of the Optical Society of America, 6, 1052-1069.

Braunstein, M. L., \& Andersen, G. J. (1986). Testing the rigidity assumption: A reply to Ullman. Perception, 15, 641-644.

Braunstein, M. L., Hoffman, D. D., \& Pollick, F. E. (1990). Discriminating rigid from nonrigid motion: Minimum points and views. Perception \& Psychophysics, 47, 205-214.

Braunstein, M. L., Hoffman, D. D., Shapiro, R. S., Andersen, G. J., \& BENNETT, B. M. (1987). Minimum points and views for the recovery of three-dimensional structure. Journal of Experimental Psychology: Human Perception \& Performance, 13, 335-343.

Doner, J. F., Lappin, J. S., \& Perfetto, G. (1984). Detection of threedimensional structure in moving optical patterns. Journal of Experimental Psychology: Human Perception \& Performance, 10, 1-11. 
Hays, W. L. (1973). Statistics for the social sciences. Plymouth and London: Clarke, Doble, \& Brendon.

KoEnderink, J. J., \& VAN DoOrn, A. J. (1987). Facts on optic flow. Biological Cybernetics, 56, 247-254.

Koenderink, J. J., \& VAN DOORN, A. J. (1991). Affine structure from motion. Journal of the Optical Society of America, 8, 377-385.

LAPPIN, J. S. (1990). Perceiving metric structure of environmental objects from motion, self-motion and stereopsis. In R. Warren \& A. H. Wertheim (Eds.), The perception and control of self-motion (pp. 541576). Hillsdale, NJ: Erlbaum.

Lappin, J. S., Doner, J. F., \& Kottas, B. L. (1980). Minimum conditions for the visual detection of structure and motion in three dimensions. Science, 209, 717-719.

LAPPIN, J. S., \& FUQUA, M. A. (1983). Accurate visual measurement of three-dimensional moving patterns. Science, 221, 480-482.

Lind, M. (in press). Perceiving motion and rigid structure from optic flow: A combined weak perspective and polar perspective approach. Perception \& Psychophysics.

Longuet-HigGins, H. C. (1981). A computer algorithm for reconstruction a scene from two projections. Nature, 293, 133-135.

Norman, J. F., Todd, J. T., PerotTI, V. J., \& Tittle, J. S. (1996). The visual perception of 3D length. Journal of Experimental Psychology: Human Perception \& Performance, 22, 173-186.

Tittle, J. S., Todd, J., Perotti, V. J., \& Norman, J. F. (1995). The sys- tematic distortion of perceived 3-D structure from motion and binocular stereopsis. Journal of Experimental Psychology: Human Perception \& Performance, 21, 663-678.

ToDD, J. T. (1985). The perception of structure from motion: Is projective correspondence of moving elements a necessary condition? Journal of Experimental Psychology: Human Perception \& Performance, 11, 689-710.

Tod, J. T., AKerstrom, R. A., Reichel, F. D., \& Hayes, W. (1988). Apparent rotation in three-dimensional space: Effects of temporal, spatial, and structural factors. Perception \& Psychophysics, 43, $179-188$.

TODD, J. T., \& Bressan, P. (1990). The perception of 3-dimensional affine structures from minimal apparent motion sequences. Perception \& Psychophysics, 48, 419-430.

TODD, J. T., \& Norman, J. F. (1991). The visual perception of smoothly curved surfaces from minimal apparent motion sequences. Perception \& Psychophysics, 50, 509-523.

UlLMAN, S. (1979). The interpretation of visual motion. Cambridge, MA: MIT Press.

Wallach, H., \& O'CONNELl, D. N. (1953). The kinetic depth effect. Journal of Experimental Psychology, 45, 205-217.

(Manuscript received July 11, 1994; revision accepted for publication October $21,1995$. 